\title{
Cyberinfrastructure: Earth Data System Sustainability
}

\author{
EarthRates $\mathrm{RCN}^{1}$ \\ ${ }^{1}$ University of Connecticut
}

May 5, 2020

Czaplewski, J., Fils, D., Goring, S., Lehnert, K., Sluyter, A., Tikoff, B., Uhen, M.D., Zaslavsky, I.. (2018) Cyberinfrastructure: Earth Data System Sustainability. EarthRates, Minneapolis, MN. https://drive. google.com/file/d/11QSnDFKD8C_vMbIVwlyWzaTaVy5lM7Xd/view?usp=sharing 